\section{Surveillance of Methicillin-Resistant Staphylococcus aureus Mupirocin Resistance in a Veterans Affairs Hospital}

To the Editor - In 2009, the US Food and Drug Administration approved intranasal mupirocin for the eradication of methicillin-resistant Staphylococcus aureus (MRSA) nasal colonization in adults. ${ }^{1}$ Subsequently, SHEA and the Infectious Diseases Society of America have issued guidelines regarding appropriateness of mupirocin use in decolonization of surgical patients. ${ }^{2}$ A summary of the recommendations of the American Society of Health-System Pharmacists for the presurgical use of intranasal mupirocin was presented by Bratzler et al., ${ }^{3}$ who discussed studies supporting the use of preoperative intranasal mupirocin in colonized patients. Recently, our orthopedic surgical group adopted those recommendations. We became concerned as to the benefit that this may have versus the untoward effect of inducing higher levels of resistance. Other Veterans Affairs hospitals have reported variable rates of MRSA mupirocin resistance (MPR). ${ }^{4,5}$ It is prudent that the American Society of HealthSystem Pharmacists recommends periodic surveillance of MPR S. aureus. ${ }^{3}$ This led us to determine our inpatient rate of MPR MRSA. MPR occurs often and the mechanisms of resistance are well characterized. ${ }^{6}$ MPR is mediated by 2 genes: a plasmid-associated gene, тирA, that imparts high-level resistance (HLR); and mutations in the chromosomal gene, ileS, responsible for low-level resistance (LLR). ${ }^{6}$

We routinely test all patient admissions for MRSA by DNA polymerase chain reaction of nasal swab samples. Patients are additionally screened upon change of hospital locations and at discharge. Active surveillance is maintained through our infection control and antibiotic stewardship programs. We evaluated 82 individual MRSA inpatients during a period of 1 year at the 100-bed Robley Rex Veterans Medical Center, in order to obtain a random sampling of MRSA isolates for mupirocin in vitro susceptibility testing. The mean (range) patient age was 66 (25-93) years. We separated our results into 2 groups. Group 1 contained 57 MRSA isolates obtained from wounds (33), bone (6), blood (3), and other tissues (15). None were from sputum or urine. Group 2 contained 25 isolates from nares swab samples with positive results for MRSA by polymerase chain reaction, recovered on MRSASelect Agar (Bio-Rad). Mupirocin testing was performed with the epsilometer test (E-test) on Mueller Hinton II agar, at $36^{\circ} \mathrm{C}$ with overnight incubation. Mupirocin minimum inhibitory concentration (MIC) interpretive criteria used for this study were chosen based upon other studies ${ }^{4,5}$ (susceptible, $\leq 4 \mu \mathrm{g} / \mathrm{mL}$; LLR, 8-256 $\mu \mathrm{g} / \mathrm{mL}$; and HLR, $\geq 512 \mu \mathrm{g} / \mathrm{mL})$.
In group 1, $4(7 \%)$ of 57 isolates were MPR (1, LLR; 3, HLR). In group 2, 6 (24\%) of 25 isolates were MPR (2, LLR; 4, HLR). Combined MPR of the 82 isolates was $12 \%$. The median (range) mupirocin MIC level for LLR was $12(12-96) \mu \mathrm{g} / \mathrm{mL}$. The HLR isolates all had mupirocin MIC levels greater than $1024 \mu \mathrm{g} / \mathrm{mL}$. Using the Fisher exact test, the difference between the number of MPR isolates in groups 1 and 2 was nonsignificant $(\mathrm{P}=0.06)$; however, the comparison of rates had a $\mathrm{P}=0.04$.

A box-and-whisker analysis of the combined and separated groups for mupirocin susceptible isolates was performed (Figure 1). Shown is the distribution of MIC values in all groups. Histogram analysis of the combined group showed the median (range) mupirocin MIC was $0.19(0.094-0.5) \mu \mathrm{g} / \mathrm{mL}$. The significant differences between the means of groups 1 and 2 were determined by the comparison of means test. The histogram mean MIC for group 1 susceptible isolates was $0.184 \mu \mathrm{g} / \mathrm{mL}$, median $0.125 \mu \mathrm{g} / \mathrm{mL}$; for group 2 isolates the mean MIC was $0.277 \mu \mathrm{g} / \mathrm{mL}$, median $0.25 \mu \mathrm{g} / \mathrm{mL}(\mathrm{P}=0.001)$. The mean MIC of isolates from the nares was significantly higher than those from tissues. This correlates with the overall higher rate of MPR found in isolates from nares swabs. Owing to the low number of samples collected for this study, we are unable to make any conclusions regarding MPR in the overall population of MRSA colonizers. However, the data suggest that for our facility, MRSA colonizers may be more MPR than those isolates obtained from surgical site infections. In this small group of patients, at least $24 \%$ would not have benefited by nasal decolonization with mupirocin. If MPR increases during repeat clinical use as others have described, ${ }^{7}$ then we expect these rates to increase.

We then sought to determine when the isolates in group 1 developed MPR. Was this owing to prior mupirocin exposure in a MRSA-colonized patient, or was this a hospital/communityacquired strain of MPR MRSA? We performed a review of patients in group 1 . We found 6 of 9 patients to be previously colonized with MRSA, prior to the first-time isolation of the organism from tissue. All patients lacked a history of mupirocin therapy. Therefore, it appears that mupirocin therapy does not necessarily precede MPR. Rather, long-term colonization with MRSA may be a risk factor for MPR.

We found only one recent study, performed in the Netherlands, that evaluated MPR in colonized patients. Bode et al. ${ }^{8}$ obtained 1251 patient nasal swab samples containing S. aureus; all isolates were susceptible to both methicillin and mupirocin. Therefore, our results may be limited to the veteran population and may be local to our facility. Nevertheless, this information will help to determine our baseline for periodic surveillance, as recommended by the American Society of Health-System Pharmacists. We share our study in the hope that others will realize the importance of reporting their surveillance studies; the goal is to determine when preoperative mupirocin therapy becomes limited in use owing to increased MPR. 


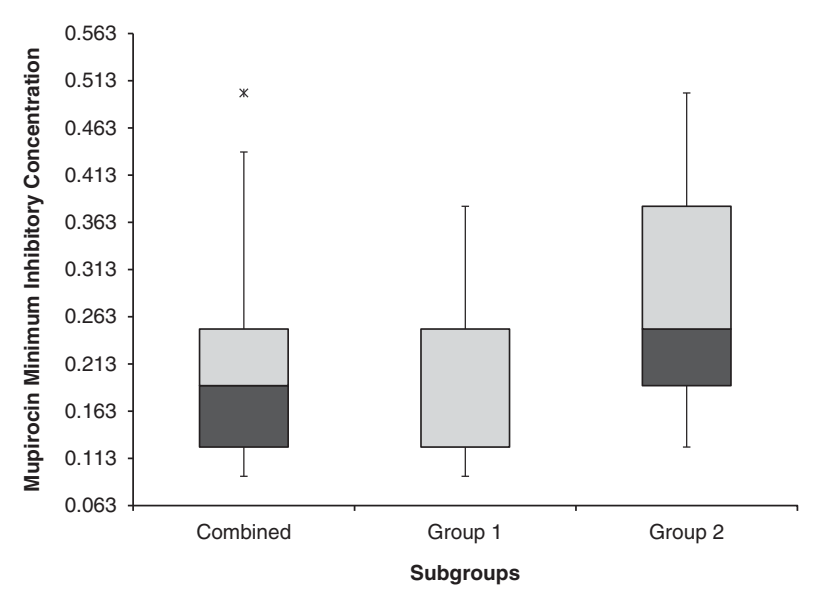

FIGURE 1. Box-and-whisker chart of mupirocin-susceptible, methicillin-resistant $S$. aureus isolates. Left to right: combined groups 1 and 2; group 1, tissue isolates; group 2, nares isolates.

\section{ACKNOW LEDGMENTS}

Financial support. This clinical study was performed at the Robley Rex Department of Veterans Affairs Medical Center, Louisville, KY, as part of our Antimicrobial Stewardship Program. All authors report no conflicts of interest relevant to this article.

\section{Carmen V. Sciortino, PhD; ${ }^{1,2}$ Mary Kemper, PhD, ASCP; ${ }^{1}$ Latha Parthasarathy, PhD, ASCP; ${ }^{1}$ James Lay, BS, MT, ASCP}

Affiliations: 1. Robley Rex Veterans Medical Center, Louisville, Kentucky; 2. Division of Infectious Diseases, University of Louisville, School of Medicine, Louisville, Kentucky.

Address correspondence to Carmen Sciortino, PhD, Robley Rex Veterans Medical Center, Pathology and Laboratory Medicine (113), 800 Zorn Avenue, Louisville, KY 40206 (Carmen.Sciortino@va.gov).

Infect Control Hosp Epidemiol 2015;36(2):235-236

(C) 2014 by The Society for Healthcare Epidemiology of America. All rights reserved. 0899-823X/2015/3602-0019. DOI: 10.1017/ice.2014.10

\section{REFERENCES}

1. Bactroban (mupirocin calcium ointment, 2\%) nasal package insert. Research Triangle Park, NC; GlaxoSmithKline; 2009 Apr.

2. Anderson DJ, Podgorny K, Berríos-Torres SI, et al. Strategies to prevent surgical site infections in acute care hospitals: 2014 update. Infect Control Hosp Epidemiol 2014;35:605-627.

3. Bratzler DW, Dellinger ED, Olsen KM, et al. Clinical practice guidelines for antimicrobial prophylaxis in surgery. Am J HealthSyst Pharm 2013;70:195-283.

4. Vasquez JE, Walker ES, Franzus BW, Overbay BK, Reagan DR, Sarubbi FA. The epidemiology of mupirocin resistance among methicillin-resistant Staphylococcus aureus at a Veterans' Affairs hospital. Infect Control Hosp Epidemiol 2000;21:459-464.

5. Babu T, Rekasius V, Parada JP, Schreckenberger P, Challapalli M. Mupirocin resistance among methicillin-resistant Staphylococcus aureus-colonized patients at admission to a tertiary care medical center. J Clin Microbiol 2009;47:2279-2280.

6. Patel JB, Gorwitz RJ, Jernigan JA. Mupirocin resistance. Clin Infect Dis 2009;49:935-941.

7. Miller MA, Dascal A, Portnoy J, Mendelson J. Development of mupirocin resistance among methicillin-resistant Staphylococcus aureus after widespread use of nasal mupirocin ointment. Infect Control Hosp Epidemiol 1996;17:811-813.

8. Bode L, Kluytmans J, Wertheim H, et al. Preventing surgical-site infections in nasal carriers of Staphylococcus aureus. N Engl J Med 2010;362:9-17.

\section{Sampling Plans for Use of Rapid Adenosine Triphosphate (ATP) Monitoring Must Overcome Variability or Suffer Statistical Invalidity}

\section{Reply to Visrodia}

To the Editor-We write with respect to the article by Visrodia et al. ${ }^{1}$ on using a commercial rapid adenosine triphosphate (ATP) device for validation of cleaning of flexible gastroendoscopes. The importance of timeliness in quality assurance testing in this device area is critical owing to the time pressures on the use of the gastroendoscopes by clinical staff involved in patient care. The work is a useful additional contribution to this growing field of use for ATP devices. ${ }^{2}$

Nonetheless, we highlight concerns with 2 aspects of the method adopted within the work by Visrodia et al. ${ }^{1}$ First, this work, like earlier references, utilizes only a single brand of rapid ATP device with acknowledged manufacturer support. The recommendations on "validated" relative light units (RLU) are entirely device specific and exclude other commercial devices. And, whilst the ATP/RLU readings in Visrodia et al. ${ }^{1}$ may seem dramatic (some $>100,000$ RLU), the work lacks evaluation of microbial presence that could anchor the study against a quantitated standard. ${ }^{3}$

Second, the work does not address any of the major published criticisms of the use of ATP systems as they are currently configured. Several authors have commented on the dangers of overstating the usefulness of these commercial ATP devices, the risks of alternative sources of ATP, the lack of correlation with specific pathogens of concern, the amount of ATP present within any particular cells or bacterial species, and the measurement variability that undermines statistical measures applied to the research. ${ }^{4-7}$

In this regard, and of specific concern in terms of method in Visrodia et al., ${ }^{1}$ is the way that ATP measurements and samples were obtained-for example, samples from the brush and flush sampling were divided into only 2 parts, with one part apparently used for a single ATP test and the other part tested for protein residues. The entire sample set of 